\title{
Conocimiento puro y cálculo infinitesimal en el neokantismo de Hermann Cohen
}

\author{
HERNÁN PRINGE \\ Consejo Nacional de Investigaciones Científicas y Técnicas \\ Universidad de Buenos Aires \\ Instituto de Filosofía, Universidad Diego Portales, Santiago, Chile \\ DOI: $10.36446 /$ rlf2020149 \\ Resumen: En este trabajo nos proponemos \\ analizar uno de los aspectos centrales de la filosofía de \\ Hermann Cohen:la relación que su Lógica del conoci- \\ miento puro establece entre el pensar puro y el cálculo \\ infinitesimal. Mostraremos que la interpretación cohe- \\ niana del cálculo se orienta a fundamentar en el pensar \\ puro todos los elementos que Kant distingue en la in- \\ tuición empírica: tanto su materia (la sensación), como \\ su forma (el tiempo y el espacio). De tal modo, mediante \\ el cálculo infinitesimal, Cohen buscará dar cuenta del \\ conocimiento sin recurrir a ninguna receptividad.
}

Palabras clave: Cálculo diferencial, conocimiento puro, espacio, tiempo, sensación. 


\title{
Pure Knowledge and Infinitesimal Calculus in Hermann Cohen's Neokantianism
}

\begin{abstract}
The aim of this paper is to analyze one of the central aspects of Hermann Cohen's philosophy: the relationship that his Logic of pure knowledge establishes between pure thought and infinitesimal calculus. We argue that Cohen's interpretation of calculus seeks to ground in pure knowledge all the elements that Kant distinguishes in empirical intuition: both its matter (sensation) and its form (time and space). In this way, by means of infinitesimal calculus, Cohen tries to account for knowledge without appealing to any receptivity.
\end{abstract}

Key-words: differential calculus, pure knowledge, space, time, sensation.

\section{Introducción}

口 nu Lógica del conocimiento puro, Hermann Cohen busca llevar

Ca cabo aquella inversión del modo de pensar que la filosofía de

Kant anuncia, pero, según Cohen, deja a mitad de camino. Si de acuerdo con el giro copernicano no puede haber objeto más que por medio del conocimiento, pues "el conocimiento produce el objeto" (Cohen 1907: 4), entonces tal producción no puede meramente consistir en la determinación de la pura forma de la objetividad en general. Por el contrario, también la materia del conocimiento debe ser producida por la espontaneidad del pensar. Así, Cohen afirma, contra lo que sostiene Kant, que en el conocimiento el pensar no se enfrenta a ningún dato, ni siquiera a un dato puro (Cohen 1914: 26-27). Lo que Kant llama dato es, en realidad, producto: un producto del pensar. La distinción kantiana entre pensar y conocer, basada precisamente en la consideración de la intuición sensible como elemento dado, es abandonada en favor de una doctrina del pensar que resulta, a la vez, una doctrina del conocimiento (Cohen 1914: 13). El pensar coheniano ya no es deudor de ninguna receptividad que le brinde un contenido no originado en el pensar mismo. Según Cohen, solo el pensar así concebido (y no el pensar tal como lo entiende Kant) puede verdaderamente ejecutar el "giro copernicano" que la crítica prescribió a la metafisica.

En este trabajo nos proponemos analizar uno de los aspectos centrales de la doctrina de Cohen: la relación que su Lógica establece entre el conocimiento puro y el cálculo infinitesimal. Mostraremos que la interpretación coheniana del cálculo se orienta a fundamentar en el pensar puro todos los 
elementos que Kant distingue en la intuición empírica: tanto su materia (la sensación), como su forma (el tiempo y el espacio). De tal modo, mediante el cálculo infinitesimal, Cohen buscará dar cuenta del conocimiento sin recurrir a ninguna receptividad. ${ }^{1}$

\title{
2. Pensar puro y cálculo infinitesimal
} Para Cohen, el pensar es puro en tanto nada se le enfrenta como
un dato y el conocimiento es puro en tanto su origen es únicamente un pensar tal. Según Cohen, Kant se equivoca al postular un elemento puro del conocimiento, que, sin embargo, no tiene su origen en el pensar. Contra Kant, Cohen sostiene que "la pureza puede ser solamente de una única clase" (Cohen 1914: 150). La intuición no puede ser pura precisamente porque es irreductible al pensar. Pero la pureza del pensar no consiste meramente en que nada le es dado, sino más bien en que él produce su propio contenido. Por lo tanto, en contraposición a la doctrina de Kant, el pensar no debe ser aplicado a ningún objeto que le resulte heterogéneo.Ya no hay lugar para la pregunta de Kant acerca de las condiciones de la aplicación de un concepto puro del entendimiento a un fenómeno sensible.Tal concepción inadecuada del pensar se revela en el esquematismo kantiano (Cohen 1914: 151). Recordemos que en dicho capítulo de la Crítica Kant sostenía lo siguiente:

\begin{abstract}
Ahora bien, conceptos puros del entendimiento son completamente heterogéneos en comparación con intuiciones empíricas ( $\mathrm{y}$ en general, con [intuiciones] sensibles), y nunca pueden ser hallados en intuición alguna. Entonces, ¿cómo es posible la subsunción de las últimas bajo los primeros, y por lo tanto, la aplicación de la categoría a fenómenos [...]? Esta pregunta tan natural e importante es, propiamente, la causa que hace necesaria una doctrina transcendental de la facultad de juzgar; a saber, para mostrar la posibilidad de cómo conceptos puros del entendimiento pueden ser aplicados a fenómenos. (Kant, KrV A137/B176 - A138/B177)
\end{abstract}

\footnotetext{
${ }^{1}$ En su reciente libro sobre Hermann Cohen, Beiser afirma que en la Lógica del conocimiento puro Cohen simplemente presenta su tesis acerca de la "autonomía" o "independencia" del pensar sin explicar cómo llega a este resultado (Beiser 2018: 196). Por el contrario, en lo que sigue intentaremos reconstruir los argumentos desarrollados en la Lógica del conocimiento puro que sostienen las tesis de Cohen.
} 
Contra Kant, Cohen rechaza toda presunta heterogeneidad entre el pensar y el ser. Mientras que Kant se esfuerza en demostrar que el pensar se aplica a fenómenos a pesar de ser un pensar puro, Cohen afirma que el pensar se aplica al objeto del conocimiento precisamente porque es un pensar puro. La pureza del pensar consiste, ni más ni menos, en su aplicabilidad.

La peculiaridad de la doctrina de Cohen es el rol central que ella le asigna al cálculo infinitesimal en la producción del ser por el pensar. ${ }^{2} \mathrm{La}$ interpretación coheniana del infinitesimal es la clave de bóveda que sostiene el edificio de la lógica del conocimiento puro. En efecto, Cohen considera que Kant no llevó a cabo una adecuada lectura del significado del cálculo para la filosofia transcendental y por ello no fue capaz de reconocer la capacidad productiva del pensar. Según Cohen, "si el principio infinitesimal hubiera encontrado en la Crítica el lugar que le corresponde, entonces la sensibilidad no habría podido ponerse delante del pensar; el pensar puro no habría sido debilitado en su autonomía" (Cohen 1914: 35). En cambio, la lógica del conocimiento puro sí reconocerá "el determinante significado lógico del principio infinitesimal" y ella será "la lógica del principio del cálculo infinitesimal" (Cohen 1914: 34), pues este proporciona el instrumento para que el pensar genere desde sí su propio contenido. Mediante el método infinitesimal el pensar produce aquella materia que, según Kant, solamente podía ser provista por la sensibilidad.

De acuerdo con la doctrina de Kant, en el conocimiento los conceptos puros del entendimiento se aplican a los fenómenos sensibles. El conjunto de aquellos juicios sintéticos que se infieren a priori de las categorías según las condiciones de aplicación a los fenómenos impuestas por el esquematismo será el sistema de todos los principios (Grundsätze) del entendimiento (Kant, KrV A136/B175). "Kant", dice Cohen, "distinguió el pensar puro de la intuición pura; por lo tanto él necesitó el enlace de ambas condiciones heterogéneas, que debió buscar en el principio (Grundsatz)" (Cohen 1914: 477). En cambio, la lógica coheniana del conocimiento será una doctrina que no contendrá elementos originados en ninguna otra fuente más que en el pensar mismo. Cohen abandonará la distinción kantiana entre "la síntesis de diversas representaciones en un juicio y la síntesis de diversas representaciones en una intuición" (Kant, KrVA79 /B104-B105). Por lo tanto, los fundamentos transcendentales del conocimiento no serán para Cohen principios (Grundsätze), sino lisa y llanamente juicios (Urteile). ${ }^{3}$ La lógica buscará mostrar que estos

${ }^{2}$ Giovanelli ofrece una exhaustiva reconstrucción histórica del método infinitesimal coheniano en Giovanelli 2016. Véase también Mormann y Katz 2013.

${ }^{3}$ Véase Edel 1988: 516-523. 
juicios del pensar puro poseen referencia objetiva por más que, a diferencia de los Grundsätze kantianos, no contengan ningún elemento alógico. ${ }^{4}$

Cohen distingue cuatro clases de juicios del pensar puro (Cohen 1914: 77-78): ${ }^{5}$ los juicios de las leyes del pensar, ${ }^{6}$ los juicios de la matemática, los juicios de la ciencia matemática de la naturaleza y los juicios de la metodología. La primera clase corresponde a los juicios de la cualidad, la segunda a los de la cantidad, la tercera a los de la relación y la última a los de la modalidad. La interpretación coheniana del cálculo diferencial e integral se desarrollará en los juicios de la matemática, es decir en los juicios de la cantidad. ${ }^{7}$ Tales juicios, valga aquí la aclaración, no son los juicios propios de la ciencia matemática, formulados por el matemático, sino más bien aquellos juicios metafisicos que constituyen el fundamento transcendental del conocimiento matemático, formulados por el filósofo. Estos juicios son los de realidad, multiplicidad y totalidad. A continuación, analizaremos el rol de cada uno de ellos en la generación del conocimiento puro.

\section{El juicio de realidad}

T a matemática es el ejemplo más claro del pensamiento puro, pero

Lno porque ella sea una ciencia independiente de la experiencia. Por el contrario, el conocimiento matemático tiene una relación necesaria con la experiencia. La pureza de la matemática no consiste en su aislamiento respecto de lo empírico, sino más bien precisamente en su aplicabilidad a él. La matemática, tal como la concibe Cohen, es aquella "de la ciencia matemática de la naturaleza" (Cohen 1914: 121). ${ }^{8}$ En la matemática se verifica de modo paradigmático la producción del ser por parte del pensar. El valor metódico del pensamiento matemático consiste en que ninguno de sus contenidos es tomado como dado: todo es generado por el pensar. Tal generación es un devenir, un tránsito del no ser al ser, que se corresponde con la noción fisica fundamental de movimiento. Los "juicios de la matemática" serán entonces los juicios que expresen el fundamento transcendental de la

\footnotetext{
${ }^{4}$ Más precisamente, como indicamos arriba, Cohen entiende que la referencia objetiva del pensar solamente es posible porque nada se le enfrenta como un dato.

${ }^{5}$ Véase Lenk 1968: 453 ss.

${ }^{6}$ Estos corresponden a lo que tradicionalmente se concibe como lógica formal, pero, además de la identidad y la contradicción, se incluye bajo este título el juicio de origen.

${ }^{7}$ Natorp cuestiona esta división de los juicios en Natorp 1986: 15-16.

${ }^{8}$ Mormann subraya este punto en Mormann 2018: 115.
} 
matemática, en tanto ella hace posible una ciencia natural del movimiento. Para ello, Cohen mostrará que dicho fundamento se encuentra en el cálculo diferencial e integral.

Cohen coincide con Kant en que el pensamiento matemático satisface las demandas de la revolución copernicana, aunque no comparte las razones que Kant esgrime en la Crítica a favor de esta tesis. De acuerdo con la doctrina de Kant, el objeto matemático es construido en la intuición pura mediante una síntesis según conceptos del entendimiento. El objeto matemático se regiría entonces por el conocimiento, pues encontraríamos a priori en el objeto solamente aquellas determinaciones puestas en él por la razón. Sin embargo, tal construcción de los conceptos matemáticos se verifica sobre una multiplicidad que, en tanto sensible, nos es dada y que establece así un límite a la espontaneidad del pensar. Por lo tanto, desde el punto de vista de Cohen, la doctrina kantiana de la construcción de conceptos no da cuenta plenamente de la inversión del modo de pensar que se verifica en la matemática. En particular, el análisis infinitesimal muestra que el conocimiento matemático no solo no es condicionado por la sensación, sino que tampoco le debe nada a la intuición, por más pura que esta pueda ser para Kant (Cohen 1914: 124). Lo crucial aquí es que esto no implica que el conocimiento matemático resulte meramente formal y vacío, sin referencia objetiva alguna, o, en el mejor de los casos, con referencia solo a objetos matemáticos, pero no fisicos. Muy por el contrario, la matemática es un conocimiento puro en el sentido de Cohen, es decir genera de manera autosuficiente su propio objeto. En tal generación se fundamentará la necesaria aplicabilidad de la matemática a la naturaleza. Cohen intentará mostrar que el primer estadio en la generación del objeto de la ciencia natural es la producción del contenido de tal conocimiento mediante la matemática y, en particular, el cálculo infinitesimal. De este modo, la aplicación fisica de este y, en general, del número estará garantizada. La potencia generadora del cálculo se revela en la noción de "número infinitesimal," tanto en la fluxión de Newton como en el infinitesimal de Leibniz (Cohen 1914:124). ${ }^{9}$ Newton y Leibniz muestran cómo el pensar hace surgir lo finito de lo infinitamente pequeño, la extensión de lo inextenso:

El infinitesimal precede a la extensión y la fundamenta: imo extensione prius, así designa Leibniz a lo infinitamente pequeño. Por lo tanto, él se funda solamente en el pensar puro y gracias a este llega a ser el fundamento de lo finito. El origen es

\footnotetext{
${ }^{9}$ Ambos son "ejemplos" del significado fundamental del juicio de origen, el primero de los juicios de las leyes del pensar.
}

HERNÁN PRINGE - Conocimiento puro y cálculo infinitesimal en el neokantismo de... I 273-288 
así el fundamento, el juicio; y no es sensación ni intuición. (Cohen 1914: 126; énfasis en el original)

En otras palabras, el pensamiento fundamental del análisis matemático es que "lo finito debe tener su origen en un no sensible" (Cohen 1914:135). De tal modo, "el cálculo infinitesimal no busca ser más que un medio, tan solo el medio adecuado, para la determinación de lo finito" (Cohen 1914: 179).

El infinitesimal lleva a cabo el paso de la cualidad a la cantidad. En tal sentido, el infinitesimal permite la generación de un algo cuantitavo desde una nada cuantitativa, aunque cualitativamente determinada. La estructura de este movimiento de la nada al algo corresponde en general a la del juicio de origen. Pero el infinitesimal contiene además un significado particular, pues la matemática de lo infinitamente pequeño prepara un contenido cuantitativamente y cualitativamente determinado, que será condición de posibilidad de una ciencia matemática de la naturaleza. Por ello, para Cohen el infinitesimal se corresponde con un tipo de juicio específico que, sin dejar de "tener parentesco con las direcciones generales del pensar", expresa sin embargo un significado independiente, que permite que la matemática resulte aplicable a los fenómenos. "Esta independencia del método infinitesimal", sostiene Cohen, "debe corresponder al tipo peculiar de juicio que nosotros destacamos como juicio de realidad (Realität)" (Cohen 1914: 128; énfasis en el original). El cálculo diferencial encuentra así, como fundamento lógico, un tipo de juicio que garantiza su aplicabilidad a la ciencia de la naturaleza, pues la realidad, que constituye la presuposición última de la fisica, es a la vez la presuposición última del cálculo.

Según Cohen, la historia del cálculo infinitesimal presenta tres problemas centrales que el concepto de infinitesimal permite resolver. ${ }^{10}$ En primer lugar, el problema geométrico de las tangentes; en segundo lugar, el problema algebraico de las series y, finalmente, el problema dinámico de la velocidad y de la aceleración. En la solución de estos problemas es posible discernir cómo "el motivo del origen" se va precisando en el "motivo de la realidad", es decir cómo la especificidad del fundamento lógico del cálculo se va revelando progresivamente. En el problema de las tangentes, el "motivo del origen" está presente en tanto el punto en el que la tangente interseca la curva es el punto generador de la curva misma. Este significado del punto es incompatible con la antigua definición, según la cual el punto es el límite de la línea. "Ahora", sostiene Cohen, "el punto significa algo diferente, algo

${ }^{10}$ Véase también Cohen 1883: \$27. 
positivo. Él ya no es solamente el fin, sino más bien el comienzo de la línea" (Cohen 1914: 129). Tal comienzo no es un comienzo cualquiera ni arbitrario. Más precisamente, no es tanto un comienzo como un origen, pues el punto contiene la ley de generación de la curva. Utilizando palabras de Natorp, "la ley puede ser pensada, intensivamente, concentrada en el punto o, extensivamente, extendida en la trayectoria” (Natorp 1910: 220). ${ }^{11} \mathrm{La}$ curva es generada a partir de su recta tangente. Pero esta generación no se verifica simplemente en el comienzo de la misma, sino a lo largo de toda la curva. La curva es generada a partir de su recta tangente en cada punto. El análisis del fundamento lógico de este hecho matemático aísla al punto generador de la curva, como si se tratara de cierto tipo de punto absoluto. "Este carácter absoluto del punto", sostiene Cohen, "en tanto a partir de él la curva se genera ininterrumpidamente, lo señalamos como realidad" (Cohen 1914: 130; énfasis en el original). En el mismo sentido, en el caso de las series infinitas, el término general representa la ley de la serie y contiene así la regla de su generación. Más notable es aún la presencia de este elemento generador en el problema dinámico. Allí, sostiene Cohen, "la exigencia de un punto absoluto tal no puede evitarse, pues solamente mediante él el movimiento real puede ser definitivamente diferenciado de una sucesión subjetiva de representaciones" (Cohen 1914: 130). Este punto, que representa a la aceleración, es el fundamento último del movimiento. Respecto de la forma del movimiento, el punto "vale como absoluto". El punto representa así "un ser en devenir" o mejor dicho "un ser para el devenir" (Cohen 1914: 130). El punto acelerado posee una unidad que "definimos como realidad" (Cohen 1914: 131).

Al igual que Kant, Cohen subraya la distinción entre realidad (Realität) y realidad efectiva (Wirklichkeit) ${ }^{12}$. Según Kant, "realidad" o cosidad significa "un algo cuyo concepto expresa ya en sí mismo un ser [...] porque solo por ella, y solo hasta donde ella alcanza, los objetos son algo (cosas)" (Kant $K r V$ A574/B603). Según Cohen, el diferencial $(d x)$ representa lo que es (das Seiende), y solamente gracias a él aquello de lo cual él es el diferencial

\footnotetext{
${ }^{11}$ Si bien en otras obras Cohen identifica el diferencial con la magnitud intensiva - por ejemplo en Cohen 1883: 15 y Cohen 1918: 544-, en la Lógica Cohen sostiene que no hay magnitud intensiva (Cohen 1914: 492). En el mismo sentido, Gawronsky afirma que el diferencial no es una magnitud (Gawronsky 1910: 97). Véase también Holzhey 1986: 251. Giovanelli parece pasar por alto esta modificación de la teoría de Cohen en Giovanelli 2011 (véase, por ejemplo, Giovanelli 2011:210).

${ }^{12}$ Para Cohen, la realidad efectiva es una "instancia de la sensación" (Cohen 1914: 128). Véase también Cohen 1914: 462. Acerca de la relación entre sensación y realidad efectiva, véase Holzhey 1986: 159-160, Dufour 2002.
} 
(x) podrá, a su vez, en general, ser (Cohen 1914: 136). Pero, mientras que para Kant realidad es "lo que en la intuición empírica corresponde a la sensación" (Kant KrVA168/B209), ${ }^{13}$ para Cohen la realidad no depende de la sensación. ${ }^{14}$ Más bien, ahora es la realidad del infinitesimal y no la sensación la condición última de que haya, en general, contenido del pensar y así conocimiento. Es el infinitesimal lo que vuelve al pensar un pensar del ser. Pero este infinitesimal tiene su origen en el pensar mismo y no en una presunta receptividad. Así, la realidad del infinitesimal presupone que nos liberemos de la función cognoscitiva de la sensación (Cohen 1914: 128). ${ }^{15}$

La categoría kantiana de realidad determinaba un ser previamente dado por la sensibilidad. Por el contrario, el juicio coheniano de realidad genera el ser como una realidad infinitesimal. Si el juicio de origen afirma que "el pensar puro debe comenzar con el origen, en tanto el pensar es pensar del conocimiento y por lo tanto del ser" (Cohen 1914: 134), el juicio de realidad sostiene que el ser así originado es representado, como realidad, por lo infinitesimal. Lo infinitesimal es el fundamento no sensible de lo finito y en este pensamiento se unen la matemática y la ciencia de la naturaleza. ${ }^{16}$ Mediante el infinitesimal, la matemática le entrega a la ciencia de la naturaleza aquello que esta busca en todas sus determinaciones: lo real. "Es por ello", sostiene Cohen, "que el concepto en el que esta entrega se verifica puede ser llamado realidad" (Cohen 1914: 135).

Según la doctrina de Kant, la espontaneidad del pensar sintetiza en una unidad la multiplicidad dada por la sensibilidad. En esta acción de unificación, el entendimiento impone sus leyes a los fenómenos, pues tal síntesis se encuentra reglada por las categorías. Por el contrario, para Cohen el pensar puro rige al objeto del conocimiento porque lo genera desde sí. En los juicios de la matemática, aquella multiplicidad espacio-temporal que Kant entendía como pasivamente recibida, es producida por el pensar y, en esta producción es, a la vez, reunida en una unidad que la vuelve apta para recibir las determinaciones de la ciencia matemática de la naturaleza. La multiplicidad y la unidad de la multiplicidad (es decir, su totalidad) serán generadas por el pensar. El fundamento de ambas será la realidad del infinite-

\footnotetext{
${ }^{13}$ Véase también Kant $\mathrm{KrV}$ A143/B182.

${ }^{14}$ Kant sostiene incluso: “Sensatio [est] realitas phaenomenon” (Kant $\mathrm{KrV}$ B186).

${ }^{15} \mathrm{Al}$ respecto, véase Flach 2009: 40.

${ }^{16} \mathrm{El}$ origen de lo finito es definido en el diferencial, pero solamente resulta determinable mediante el cociente diferencial (Cohen 1914: 182). Giovanelli sostiene que aquí Cohen rechaza explícitamente la prioridad del conciente diferencial sobre el diferencial (Giovanelli 2016: 18). Moynahan sigue la interpretación de Giovanelli en Moynahan 2018: 60.
} 
simal. En tal sentido, Cohen sostiene que "la verdadera unidad consiste en lo infinitesimal" (Cohen 1914: 137). El diferencial es la unidad primigenia que fundamenta la multiplicidad. Con ello, la realidad infinitesimal será el fundamento del número. Pero en la generación del número también participarán otras operaciones del pensar. En particular, el pensar generará el número mediante la producción de una multiplicidad. Con esta acción peculiar, el pensar generará también el tiempo. La multiplicidad así producida será a su vez reunida en una totalidad representada por la integral. Esta totalidad será el origen del espacio. El cálculo diferencial e integral expresará entonces el modo en el que el pensar puro genera el contenido de la ciencia matemática de la naturaleza, es decir, para Cohen, el contenido de la experiencia. Veremos ahora cómo esta generación se verifica.

\section{El juicio de multiplicidad}

Cl origen puro del número se encuentra en la unidad primigenia mediante la acción representada por el juicio correspondiente. Cohen distingue esta multiplicidad (Mehrheit) de la pluralidad (Vielheit) de un conjunto de elementos que preceden a la operación originaria del pensar. Los elementos de una pluralidad le son dados al pensar, mientras que los de una multiplicidad son producidos por el pensar puro. Más precisamente, la multiplicidad es el resultado de un adicionar que no consiste en la reunión de elementos lógicamente previos a tal adición: "El sumar mismo, en el que los sumandos en primer lugar surgen, constituye la tarea; y su posiblidad es el problema" (Cohen 1914: 149)

El juicio de realidad genera la unidad absoluta, que Cohen representa con el 1. Esta unidad es un puro ser, sin otra determinación. Es el ser absoluto, que es presupuesto por todo ser determinado, pero que no lo agota. A él, Cohen lo llama "el número de la realidad". El juicio de multiplicidad, por el contrario, genera "el número de la multiplicidad". Él es la unidad entendida como término de una serie. Tal unidad puede ser representada por la variable $x$, o como un $A$ determinado, cuya única determinación es ser un término tal. El juicio de multiplicidad expresa esta acción de seriación mediante la cual el pensar puro genera los términos de la serie a partir de su término general. Estos términos son así puestos por el pensar y no le son dados a él.

En esta seriación se encuentra el fundamento del tiempo, que ya no será, como para Kant, una intuición pura, sino más bien una categoría. De acuerdo con la doctrina de Cohen, el modo fundamental del tiempo no es la secuencia o sucesión (Folge). Según la sucesión se ordenan representaciones 
independientes de este orden. La temporalidad es en este caso meramente su forma. Respecto de la sucesión, afirma Cohen: "El error se halla, empero, más profundamente, en que en este modo de determinación del tiempo, las representaciones mismas ya están ahí, y solo resta determinar su forma, su ordenamiento temporal" (Cohen 1914: 153).

La sucesión temporal es incapaz de satisfacer la demanda del pensar puro coheniano, pues tal sucesión refleja el carácter dado de lo que así se ordena.Y, Cohen sostiene, tal carácter "pone en peligro a la pureza" (Cohen 1914: 151). Por el contrario, el acto del pensar que genera la temporalidad es la anticipación. Este es el fundamento puro del tiempo. El tiempo, sostiene Cohen, "es la categoría de la anticipación" (Cohen 1914: 155). Mientras que la sucesión "mira hacia atrás", en tanto ordena lo que ya es, la serie de la anticipación, "mira hacia delante" generando aquello que sigue como un algo que debe seguir."En vista del todavía no surge el ya no," dice Cohen. "Así surge la primera forma de la multiplicidad: en la diferenciación del pasado respecto del acto originario del futuro" (Cohen 1914: 155). Este acto de anticipación no se relaciona con lo anticipado como una forma impuesta a una materia de cuyo origen no puede dar cuenta, tal como sucede, según Kant, con el tiempo en tanto forma de la sensibilidad. Más bien, el acto como forma genera la materia, o en términos de Cohen, "la generación misma es lo generado". A partir del acto de seriación se genera un contenido determinado $A$ que no es sino el punto respecto del cual se verifica la anticipación. Toda su determinación se reduce a ser un punto tal. El futuro genera así el pasado. El tiempo como categoría "no tiene la tarea ni la competencia de generar otro tipo de contenido más que esta correlación de futuro y pasado" (Cohen 1914: 156). Así, sostiene Cohen, "la categoría del tiempo ha generado las unidades de la multiplicidad y con ello ha generado en el pensar puro el contenido que, de no ser de este modo, vale como dado" (Cohen 1914: 193). En otras palabras, según la doctrina de Cohen, el pensar puro genera, mediante la operación de anticipación, aquella multiplicidad temporal que la filosofia de Kant consideraba pasivamente recibida por el sujeto.

\section{El juicio de totalidad}

M ientras que el juicio de realidad pone la unidad absoluta de Vlo infinitesimal, el juicio de multiplicidad pone a tal infinitesimal como término de una serie temporal. El ser carente de determinación, representado por el infinitesimal, es ahora determinado como un ser desplegado en la multiplicidad del tiempo, que recibe su determinación de la anticipación realizada por el pensar puro. Ahora bien, con ello todavía 
el contenido del pensar no ha adquirido la determinación requerida por la ciencia matemática de la naturaleza: la multiplicidad de la serie temporal aún debe ser unificada. Esta terminación de la serie es el producto de una nueva operación del pensar, correspondiente al juicio de totalidad. El juicio de totalidad realizará el enlace entre la infinitud de la serie y el carácter infinitesimal de sus términos. La totalidad de la serie será la reunión de sus infinitos términos infinitesimales. Esta reunión es la representada mediante la operación matemática de integración. "La integral", sostiene Cohen, "no es sino la totalidad en la que la serie infinita se enlaza con lo infinitesimal" (Cohen 1914: 182). De tal modo, Cohen afirma que solamente desde un punto de vista formal es admisible considerar al cálculo integral como el inverso del cálculo diferencial. Más bien, es el cálculo integral aquel que en definitiva brinda sentido al cálculo diferencial, porque la invención de lo infinitesimal tuvo el propósito último de determinar lo finito mediante su representación como suma infinita de infinitesimales. "Por lo tanto", subraya Cohen, "la totalidad es el verdadero fin y el verdadero asunto del infinitesimal" (Cohen 1914: 183).

La integración llevará a cabo aquella unificación de la multiplicidad necesaria para alcanzar el contenido de la ciencia de la naturaleza. Pero para ello, se requerirá la consideración de una nueva categoría, adicional al número y al tiempo. Tal categoría es el espacio. "El ser debe volverse algo externo (ein Aussen) para el pensar", sostiene Cohen. "Esto no lesiona la identidad, pues el pensar mismo genera esto externo; y mediante esta generación el pensar se vuelve pensar de la naturaleza, por lo tanto ser" (Cohen 1914: 188; énfasis en el original). Tal como sucedió con el tiempo, Cohen rechaza así el carácter dado que Kant le atribuye al espacio. Más bien, en el juicio de totalidad, el espacio se realiza como categoría. La multiplicidad temporal es la de una serie que no puede ser pensada como línea. En ella solamente se verifican anticipaciones, ella es "un infatigable ir y venir, un tender hacia delante y mirar hacia atrás" (Cohen 1914: 193). Pero nada permanece, más allá de este monótono modo mismo de generación. El flujo temporal incesante impide que se constituya un contenido permanente. Para ello, es necesaria la coexistencia (Beisammen). Tal es la nueva tarea requerida para la posibilidad de la experiencia, que será llevada a cabo por el espacio (Cohen 1914: 194). Como en una adición infinita, los infinitos elementos serán reunidos en la totalidad del espacio. La espacialidad no es entonces, como la intuición pura de Kant, dada sino, por el contrario, producida por el pensar. La coexistencia misma, puesta por el pensar mediante el juicio de totalidad, es sin más la exterioridad. La conservación de lo coexistente es el volverse exterior (Cohen 1914: 196). Tal como el tiempo, el espacio no es condición de posibilidad del darse de los fenómenos, y por lo tanto una intuición pura, sino que es un 
instrumento del que el pensar puro se vale para la generación de la naturaleza y resulta así una categoría del pensar (Cohen 1914: 197).

Si consideramos una vez más el problema dinámico, la realidad infinitesimal corresponde a la de un móvil con cierta velocidad instantánea, que el pensar puro le asigna según el juicio de realidad. El juicio de multiplicidad genera la serie temporal de las velocidades instantáneas. El juicio de totalidad reunirá estas anticipaciones en una unidad, que será espacial. Más precisamente, la acción de tal juicio será la integración de las velocidades instantáneas en el intervalo temporal anticipado. El resultado será la trayectoria del móvil, es decir el espacio recorrido. Así, Cohen afirma:

Mediante las secciones cónicas, los planetas mismos, los contenidos más generales, se vuelven determinables. También en ellas se encuentra la presuposición de la totalidad.Y en su cómputo ha surgido el cálculo infinitesimal. La integración de la ecuación es la integración de la curva. Así el cálculo integral presenta como su fin la determinación del espacio. La diversidad que se deja determinar en las curvas significa sobre todo la diversidad de contenido que puede ser definida para la ciencia. La totalidad infinita es por lo tanto aquella que metódicamente lleva la diversidad de contenido a su determinación fundamental, mediante la integral y su significado como espacio. (Cohen 1914: 198) ${ }^{17}$

En resumen, según la doctrina de Cohen, los juicios matemáticos generan el contenido de la naturaleza y proporcionan así las condiciones de una ciencia natural matemática. El juicio de realidad genera la realidad infinitesimal. El juicio de multiplicidad, la serie temporal de esas realidades. El juicio de totalidad, la reunión de las mismas en el espacio. Esta generación del contenido se verifica mediante el cálculo diferencial e integral. Parafraseando a Galileo, Cohen sostiene que los juicios de la cantidad generan los caracteres "con los que la filosofia se escribe en el libro de la naturaleza" (Cohen 1914: 207). La lógica del conocimiento puro, es decir la filosofia, determina mediante estos juicios a la naturaleza como una realidad matematizable. Dichos caracteres deberán todavía ser enlazados en frases, para lo que se requerirá una sintaxis adecuada: "Una vez que los juicios de la matemática han creado el alfabeto sobre la base de los juicios de las leyes del pensar, los juicios de la ciencia matemática de la naturaleza se incorporan al plan, para escribir la filosofia de la naturaleza con esas letras" (Cohen 1914: 588).

${ }^{17}$ Véase Cohen 1883: $₫ 91, \S 28$. 
Las reglas sintácticas según las que se construirán frases usando el alfabeto matemático serán provistas por los juicios de la relación, denominados juicios de la ciencia natural matemática. Lamentablemente, la discusión de estos juicios se encuentra fuera de los límites de este estudio y deberemos dejarla para otra oportunidad.

\section{Conclusiones}

Tos juicios de la matemática establecen cómo todos los elementos Lde lo que Kant llama "intuición empírica" encuentran su fundamento en el pensar puro. Por un lado, aquel contenido que Kant llama materia de la intuición empírica ya no es caracterizado como sensación, es decir como el efecto de una afección. Tal contenido es, para Cohen, la realidad infinitesimal, que no es pasivamente recibida sino espontáneamente producida por el pensar en el juicio de realidad. Por otro lado, el tiempo y el espacio, las formas kantianas de la intuición, tampoco son dados. Más bien, tiempo y espacio son generados mediante los juicios de multiplicidad y de totalidad respectivamente. Tal generación produce, además, la categoría de número. Los juicios de realidad, multiplicidad y totalidad fundamentan al cálculo infinitesimal como el modo en el que el pensar puro genera el contenido de la experiencia, mostrando que el conocimiento no solamente rige la forma de su objeto sino también su materia. Mediante esta interpretación del cálculo infinitesimal, Cohen busca llevar la inversión kantiana del modo de pensar más allá del estadio alcanzado por el propio Kant. "Nosotros comenzamos con el pensar" dirá Cohen. Es decir, la lógica coheniana no procede, como la Crítica de la razón pura, partiendo de la sensibilidad. "El pensar no puede tener ningún origen fuera de sí mismo, si su pureza no ha de ser restringida ni empañada. El pensar puro en sí mismo, con exclusión de toda otra cosa, debe generar exclusivamente los conocimientos puros" (Cohen 1914: 13). El cálculo infinitesimal, según Cohen, hace posible esta tarea. ${ }^{18}$

\footnotetext{
${ }^{18}$ Este trabajo ha sido financiado por el programa Horizon 2020 de la Unión Europea, mediante el subsidio Marie Skłodowska-Curie $\mathrm{N}^{\circ}$ 777786. La investigación también es parte de los proyectos CONICYT/FONDECYT Regular No 1190965 y PR65/19-22446 (Comunidad de Madrid y Universidad Complutense de Madrid).

The project leading to this paper has received funding from the European Union's Horizon 2020 research and innovation programme under the Marie Skłodowska-Curie grant agreement $\mathrm{N}^{\circ}$ 777786. The investigation is also part of the projects CONICYT/FONDECYT Regular N 1190965 and PR65/19-22446 (Comunidad de Madrid and Universidad Complutense de Madrid).
} 


\section{BIBLIOGRAFÍA}

Beiser, F. (2018), Hermann Cohen: an Intellectual Biography (Oxford: Oxford University Press).

Cammarota, G. P. (2009) (ed.), Unità della ragione e modi dell'esperienza: Hermann Cohen e il neokantismo (Soveria Mannelli: Rubbettino).

Cohen, H. (1883), Das Princip der Infinitesmal-Methode und seine Geschichte: ein Kapitel zur Grundlegung der Erkenntnisskritik (Berlín: Dümmler).

Cohen, H. (1907), Kommentar zu Immanuel Kants Kritik der reinen Vernunft (Leipzig: Meiner).

Cohen, H. (1914), Logik der reinen Erkenntnis. System Der Philosophie, 1 (Berlín: Bruno Cassirer).

Cohen, H. (1918), Kants Theorie der Erfahrung (Berlín: Bruno Cassirer).

Damböck, Ch. (2018) (ed.), Philosophie und Wissenschaft bei Hermann Cohen (Cham: Springer).

Dufour, E. (2002), "Le statut du singulier: Kant et le néokantisme de l'École de Marbourg", Kant-Studien, 93 (3): 324-350.

Edel, G. (1988), Von der Vernunftkritik zur Erkenntnislogik (Freiburg - München: Alber).

Flach, W. (2009), "Hermann Cohens systemtheoretische Subjektslehre”, en G. P. Cammarota (ed.), Unità della ragione e modi dell'esperienza: Hermann Cohen e il neokantismo (Soveria Mannelli: Rubbettino, 35-45).

Giovanelli, M. (2011), Reality and Negation: Kant's Principle of Anticipations of Perception (Dordrecht: Springer).

Giovanelli, M. (2016), "Hermann Cohen's Das Princip der Infinitesimal-Methode: the History of an Unsuccessful Book", Studies in History and Philosophy of Science, 58: 9-23.

Gawronsky, D. (1910), Das Urteil der Realität und seine mathematischen Voraussetzungen. Diss. Marburg (Weimar: Hof Buchdruckerei).

Holzhey, H. (1986), Cohen und Natorp, 2 vol. (Basel - Stuttgart: Schwabe).

Kant, I. (KrV), Crítica de la razón pura, estudio preliminar, traducción y notas de M. Caimi (Buenos Aires: Colihue, 2007) (citado indicando edición A/B y número de página).

Lenk, H. (1968), Kritik der logischen Konstanten (Berlín: de Gruyter).

Mormann, Th. (2018), "Zur Mathematischen Wissenschaftsphilosophie des Marburger Neukantianismus”, en Ch. Damböck (ed.), Philosophie und Wissenschaft bei Hermann Cohen (Cham: Springer, 101-134).

Mormann, Th. y Katz, M. (2013), "Infinitesimals as an Issue of Neo-Kantian Philosophy of Science", Hopos: The Journal of the International Society for the History of Philosophy of Science, 3 (2): 236-280.

Moynahan, G. B. (2018), "The Challenge of Psychology in the Development of Cohen's System of Philosophy and the Marburg School Project," en Ch. Damböck (ed.), Philosophie und Wissenschaft bei Hermann Cohen (Cham: Springer, 41-76) 
Natorp, P. (1910), Die logischen Grundlagen der exakten Wissenschaften (Leipzig - Berlín: Teubner).

Natorp, P. (1986), “Zu Cohens Logik”, en H. Holzhey, Cohen und Natorp, 2 vol. (Basel - Stuttgart: Schwabe, 1986, v. 2, 6-40).

Recibido: 07-08-2019; aceptado: 17-03-2020 